\title{
Backbone Hydrogen Bond Strengths Can Vary Widely in Transmembrane Helices
}

\author{
Zheng Cao, ${ }^{\dagger, \S}$ James M. Hutchison, ${ }^{\dagger}$ Charles R. Sanders, ${ }^{\ddagger}$ and James U. Bowie* ${ }^{* \dagger}$ \\ ${ }^{\dagger}$ Department of Chemistry and Biochemistry, UCLA-DOE Institute, Molecular Biology Institute, University of California, Los \\ Angeles, California 90095, United States \\ ${ }^{\ddagger}$ Department of Biochemistry and Center for Structural Biology, Vanderbilt University, Nashville, Tennessee 37240, United States
}

Supporting Information

ABSTRACT: Although backbone hydrogen bonds in transmembrane (TM) helices have the potential to be very strong due to the low dielectric and low water environment of the membrane, their strength has never been assessed experimentally. Moreover, variations in hydrogen bond strength might be necessary to facilitate the TM helix breaking and bending that is often needed to satisfy functional imperatives. Here we employed equilibrium hydrogen/deuterium fractionation factors to measure backbone hydrogen bond strengths in the TM helix of the amyloid precursor protein (APP). We find an enormous range of hydrogen bond free energies, with some weaker than water-water hydrogen bonds and some over $6 \mathrm{kcal} / \mathrm{mol}$ stronger than water-water hydrogen bonds. We find that weak hydrogen bonds are at or near preferred $\gamma$-secretase cleavage sites, suggesting that the sequence of APP and possibly other cleaved TM helices may be designed, in part, to make their backbones accessible for cleavage. The finding that hydrogen bond strengths in a TM helix can vary widely has implications for membrane protein function, dynamics, evolution, and design.

\section{INTRODUCTION}

The apolar membrane creates an ideal environment for making strong hydrogen bonds. Nevertheless, direct measurements of hydrogen bonds made between amino acid side chains in membrane proteins indicate that their full potential strength is rarely realized, presumably because side-chain flexibility and water penetration into membrane proteins afford many opportunities for competing hydrogen bonds. ${ }^{1-10}$ However, backbone hydrogen bonds might be generally stronger than side-chain hydrogen bonds because there are fewer alternative hydrogen bonding partners. On the other hand, strong backbone hydrogen bonds would make it difficult to create the many transmembrane (TM) helix distortions and breaks $^{11-13}$ often required for function. ${ }^{14-18}$ We previously argued that one important mechanism for generating TM helix distortions is through the formation of alternative backbone hydrogen bonds, i.e., shifts from canonical $i+4 \alpha$-helical hydrogen bonds to $i+3$ or $i+5$ hydrogen bonds, thereby maintaining hydrogen bonding, yet allowing for conformational flexibility. ${ }^{19}$ It also remains possible that that the sequences evolved to optimize hydrogen bond strengths, sometimes to create weak points in TM helices as needed to satisfy functional requirements.

To test the possibility that backbone hydrogen bond strengths might be variable we sought a direct way to measure backbone hydrogen bond free energies. While the strength of side-chain hydrogen bonds can be probed by site-directed mutagenesis, ${ }^{1,9,10,20-34}$ this approach is not possible for backbone hydrogen bonds. Introducing amide-to-ester backbone changes into proteins via chemical synthesis provides an alternative way to address this issue, ${ }^{35-38}$ but requires thermodynamic stability measurements relative to a reference state where hydrogen bonds are broken, which is difficult to achieve for TM helices. A non-perturbing method for assessing hydrogen bond strength is to use equilibrium $H / D$ fractionation factors ( $\phi$-values) by measuring the following equilibrium:

$$
\frac{1}{2} \mathrm{D}_{2} \mathrm{O}+\mathrm{N}-\mathrm{H} \cdots \mathrm{O}=\mathrm{C} \rightleftharpoons \frac{1}{2} \mathrm{H}_{2} \mathrm{O}+\mathrm{N}-\mathrm{D} \cdots \mathrm{O}=\mathrm{C}
$$

The weaker the hydrogen bond, the stronger the preference for $\mathrm{D}$ over $\mathrm{H}^{39-52}$ The preference for $\mathrm{D}$ over $\mathrm{H}$ with reference to that in water is defined by the equilibrium constant or $\phi$ value:

$$
\phi=\frac{[\mathrm{N}-\mathrm{D} \cdots \mathrm{O}=\mathrm{C}] /[\mathrm{N}-\mathrm{H} \cdots \mathrm{O}=\mathrm{C}]}{[\mathrm{D}]_{\mathrm{w}} /[\mathrm{H}]_{\mathrm{w}}}
$$

where $[\mathrm{D}]_{\mathrm{w}}$ and $[\mathrm{H}]_{\mathrm{w}}$ are the concentrations of deuterons and protons in water.

Received: May 10, 2017

Published: July 10, 2017 
We previously placed $\phi$-values on an energetic scale by developing a semiempirical relationship between $\phi$ and the free energy required for breaking hydrogen bonds $\left(\Delta G^{\mathrm{HB}}\right){ }^{39}$ We obtained a scale factor (SF), defined as $\mathrm{SF}=\partial\left(\Delta G^{\mathrm{HB}}\right) /$ $\partial(R T \ln \phi)$, that relates the two parameters. We determined this scale factor to be $-7.0 \pm 0.7 .^{39}$ Thus, $\phi$-value measurements allow the determination of relative hydrogen bond strengths.

We decided to focus on the TM helix of the amyloid precursor protein (APP) where there may be functional reasons to create weak points in the backbone. The plaques in the brains of Alzheimer's disease patients ${ }^{53,54}$ are formed by the aggregation of short amyloid-beta $(\mathrm{A} \beta)$ peptides that are generated by sequential proteolysis of the membrane-anchored APP. ${ }^{55-57}$ Proteolysis by $\beta$-secretase first releases the ectodomain from the APP, producing the 99-residue Cterminal domain (C99 or $\left.\mathrm{APP}_{672-770}\right)$, a single-span $\mathrm{TM}$ protein. The structure of monomeric C99 in lyso-myristoylphosphatidylglycerol (LMPC) micelles derived from NMR constraints $^{58}$ is schematically represented in Figure 1. The TM

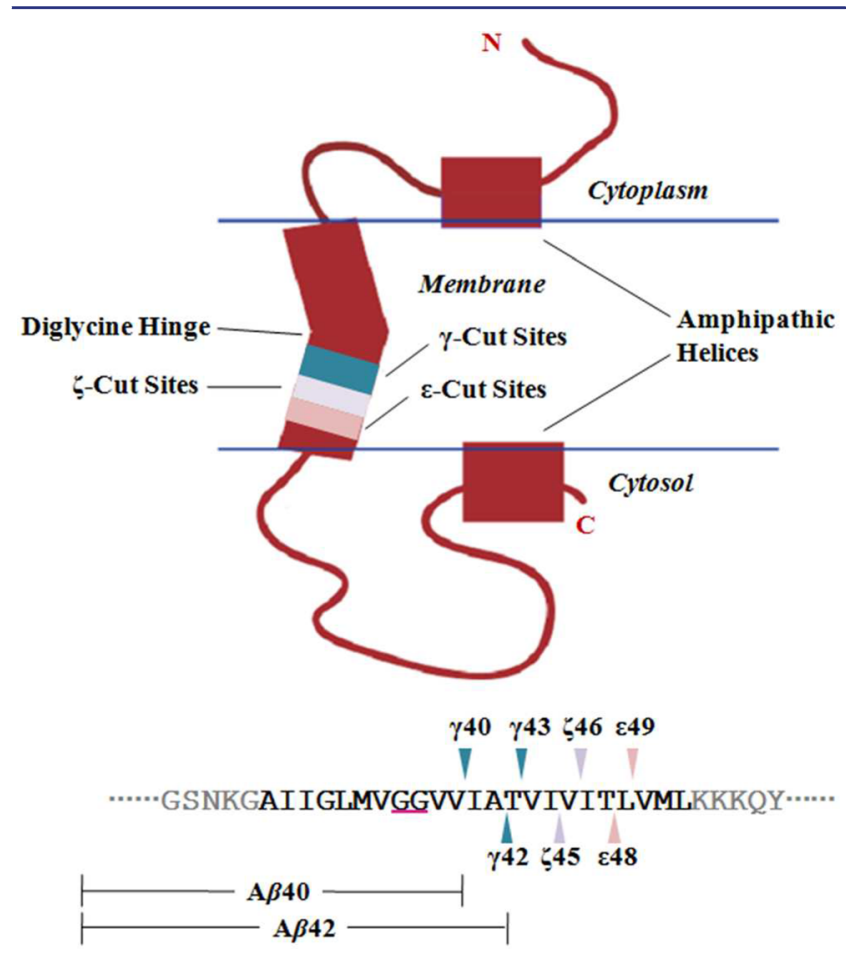

Figure 1. C99 structure and cleavage sites. Upper: Illustration of the backbone structure of $\mathrm{C} 99$ (residues 12-57 only) determined via solution-state NMR (PDB code: 2LP1). The locations of the $\varepsilon$-, $\zeta$-, and $\gamma$-cut sites are colored in pink, magenta, and cyan, respectively. Lower: Amino acid sequence of the transmembrane helix of C99 (residues 30-52). The arrows point toward the scissile bonds, using the same color coding as in the structure cartoon. The diglycine hinge is underlined in the amino acid sequence in purple. Residues outside the TM helix are colored in gray. The two primary cleavage products $\mathrm{A} \beta 40$ and $\mathrm{A} \beta 42$ are depicted under the sequence.

domain is bent at a kink around a diglycine hinge (G37, G38). Cleavage of $\mathrm{C} 99$ occurs processively C-terminal to the diglycine hinge, usually in three residue steps. The primary cleavage pathways are $\mathrm{A} \beta 48 \rightarrow \mathrm{A} \beta 45 \rightarrow \mathrm{A} \beta 42$ and $\mathrm{A} \beta 49 \rightarrow \mathrm{A} \beta 46 \rightarrow$ $\mathrm{A} \beta 43 \rightarrow \mathrm{A} \beta 40$, yielding the major final fragments $\mathrm{A} \beta 42$ and $\mathrm{A} \beta 40$. As $\mathrm{A} \beta 42$ is more toxic than $\mathrm{A} \beta 40$, the pattern of cleavage has important disease implications. While full-length $C 99^{59,60}$ and derived TM domain-containing fragments ${ }^{61-65}$ form homodimers under some conditions, it appears that $\gamma$-secretase binds and cleaves only the monomeric form of C99. ${ }^{66-69}$

A number of mechanisms have been suggested to contribute to the $\gamma$-secretase cleavage of $\mathrm{C} 99$, including (1) recognition of the N-terminal half of the C99 TM helix, preceding the diglycine hinge; ${ }^{59,70-72}$ (2) flexibility of the diglycine hinge $^{72-74}$ allowing entry of the C-terminal end of the C99 TM helix into the mouth of the $\gamma$-secretase active site; (3) an intrinsic propensity of the helix to break ${ }^{62}$ since helices need to unfold at least around their cleavage sites before proteolysis; ${ }^{75}$ (4) effects of the membrane environment on recognition, dynamics, and conformation of the helix; $;^{72,76-80}$ (5) conformational changes in $\gamma$-secretase for binding the helix; ${ }^{80-82}$ and (6) a three-residue recognition site within $\gamma$-secretase for sequential cleavage of the helix. ${ }^{83}$

Since hydrogen bonding is one of the main interactions that stabilize helices ${ }^{2-4,84-88}$ and backbone hydrogen bonds have to be broken during helix unwinding, the strength of backbone hydrogen bonds may be an important determinant of cleavage site preference. Somewhat counter-intuitively, the C-terminal half of the TM domain (where cleavage occurs)was found to be less flexible than the N-terminal domain based on hydrogen/ deuterium (H/D) exchange rate measurements and molecular dynamics simulations. ${ }^{74}$ Nevertheless, fraying at the C-terminal end near the initial cut site was observed, likely favoring the initial cleavage event, a result substantiated by the finding that C-terminal extensions inhibited $\varepsilon$-site cleavage. ${ }^{62}$

NMR spectroscopy has been used to measure $\phi$-values of hydrogen bonds in DNA and water-soluble proteins ${ }^{42-51}$ by allowing the $\mathrm{H} / \mathrm{D}$ exchange reaction to come to equilibrium. In the case of C99, however, we found that the half-life of H/D exchange for many amides in the TM helix are in the scale of weeks or more at $45{ }^{\circ} \mathrm{C}$ and would take too long to reach equilibrium. We therefore adopted an alternative approach to obtain the $\phi$-values by measuring forward and reverse exchange rate constants. Since $\phi$-values are the equilibrium constant of reaction [1], they can be calculated as $\phi=k(\mathrm{H} \rightarrow \mathrm{D}) / k(\mathrm{D} \rightarrow \mathrm{H})$, where $k(\mathrm{H} \rightarrow \mathrm{D})$ and $k(\mathrm{D} \rightarrow \mathrm{H})$ are the overall rate constants for $\mathrm{H}$-to-D exchange and $\mathrm{D}$-to-H exchange, respectively. Using this method, it is not necessary to wait until equilibrium has been achieved. Exchange rates in a TM helix will be a complex function of not only hydrogen bond strength but also flexibility, water accessibility, and unknown hydroxide concentrations, yet these factors cancel in the equilibrium $\phi$-value calculation. The approach yields $\phi$-values regardless of whether exchange proceeds by the EX1 or EX2 mechanism as long as all exchange reactions being compared proceed via the same mechanism. It can be concluded, however, from the discussion in Pester et al. ${ }^{73}$ that hydrogen exchange in the C99 TM helix proceeds by the EX2 mechanism.

\section{MATERIALS AND METHODS}

Protein Preparation. To measure amide $k(\mathrm{H} \rightarrow \mathrm{D})$ and $k(\mathrm{D} \rightarrow \mathrm{H})$ using NMR spectroscopy, we expressed and purified C-terminally 6 XHis-tagged C99 following either ${ }^{15} \mathrm{~N}$ uniform labeling ( $\left.\mathrm{U}_{-}{ }^{15} \mathrm{~N}-\mathrm{C} 99\right)$ or selective ${ }^{15} \mathrm{~N}$-labeling at glycine, leucine, valine, and threonine (GLVT ${ }^{15} \mathrm{~N}-\mathrm{C} 99$ ). The protocol was similar to those used before, ${ }^{1-3}$ but ${ }^{15} \mathrm{~N}$-labeled glycine, leucine, valine, and threonine and $\left[{ }^{14} \mathrm{~N}\right]$ ammonium chloride were used in the culture media as the nitrogen source for expressing GLVT- ${ }^{15} \mathrm{~N}-\mathrm{C} 99$. After purification, the samples were concentrated to produce samples containing $9 \%(\mathrm{w} / \mathrm{v})$ lysomyristoylphosphatidylglycerol (LMPG, Anatrace), $25 \mathrm{mM}$ imidazole, $20 \mathrm{mM}$ sodium acetate, $100 \mathrm{mM} \mathrm{NaCl}$, and $1 \mathrm{mM} \mathrm{Na}_{2} \cdot \operatorname{EDTA}(\mathrm{pH}$ 
6.5). The concentrated samples were first used for collecting the H-to$\mathrm{D}$ exchange data. The samples were then flash-frozen using liquid nitrogen and stored at $-20{ }^{\circ} \mathrm{C}$ until collection of D-to-H exchange data (see below).

Measurement of Exchange Rate Constants. For the H-to-D exchange reactions, buffer exchange was accomplished by two rounds of dilution and concentration at room temperature. In the first round, a $0.5 \mathrm{~mL}$ sample including $0.39 \mathrm{mM} \mathrm{U}{ }^{15} \mathrm{~N}-\mathrm{C} 99$ or $0.25 \mathrm{mM}$ GLVT- ${ }^{15} \mathrm{~N}$-C99 and $5 \% \mathrm{D}_{2} \mathrm{O}$ was mixed with a 9 -fold volume of the $\mathrm{D}_{2} \mathrm{O}$ dilution buffer at $\mathrm{pH}$ 6.5. The $\mathrm{D}_{2} \mathrm{O}$ dilution buffer included 99.7\% (v/v) $\mathrm{D}_{2} \mathrm{O}$ and matched the components of the original buffer minus LMPG. The sample was then concentrated to between 0.5 and $1.6 \mathrm{~mL}$ using a $10 \mathrm{kDa}$ MWCO centrifugal concentrators (Millipore Amicon) at $1000 \mathrm{~g}$. Next, the sample was diluted 1- to 5-fold with the $\mathrm{D}_{2} \mathrm{O}$ dilution buffer, followed by concentration to $\sim 0.5 \mathrm{~mL}$ so that the protein concentration was the same as the initial value. In the final buffer-exchanged samples, $\mathrm{D}_{2} \mathrm{O}$ concentrations were all $96-98 \%(\mathrm{v} /$ v).

For the D-to-H exchanges, the buffer exchange was accomplished after only one round of dilution and concentration at room temperature. A $0.5 \mathrm{~mL}$ sample including $0.39 \mathrm{mM} \mathrm{U}-{ }^{15} \mathrm{~N}-\mathrm{C} 99$ or $0.25 \mathrm{mM}$ GLVT $-{ }^{15} \mathrm{~N}-\mathrm{C} 99$ and $96-98 \%(\mathrm{v} / \mathrm{v}) \mathrm{D}_{2} \mathrm{O}$ recycled from the $\mathrm{H}$-to-D exchange experiments was thawed in a water bath at room temperature and mixed with a 9-fold volume of the $\mathrm{H}_{2} \mathrm{O}$ dilution buffer at $\mathrm{pH}$ 6.5. The $\mathrm{H}_{2} \mathrm{O}$ dilution buffer included $\mathrm{H}_{2} \mathrm{O}$ and matched the components of the original buffer minus LMPG. The sample was then concentrated to $\sim 0.5 \mathrm{~mL}$, leaving the protein concentration the same as its initial value. In the final buffer-exchanged samples, $\mathrm{D}_{2} \mathrm{O}$ concentrations were all $10 \%(\mathrm{v} / \mathrm{v})$.

During the buffer exchange reactions for both the H-to-D and D-to$\mathrm{H}$ exchange, the protein concentration was determined by measuring the protein concentration by absorbance at $280 \mathrm{~nm}$, using a Nanodrop UV-vis absorbance spectrometer. No LMPG was found in the flowthrough by 1 -dimensional ${ }^{13} \mathrm{C}$ NMR. Since the LMPG-to-protein molar ratio did not change during the buffer exchanges, the LMPG concentration was also the same as its initial value.

Soon after buffer exchange, samples were transferred into NMR tubes with a sealed capillary placed in the center, coaxially aligned with the NMR tubes. The sealed capillary contained $1 \mathrm{mM}{ }^{15} \mathrm{~N}$-labled $\mathrm{N}$ acetylglycine solution in $\mathrm{H}_{2} \mathrm{O}$ and served as an "external" reference to normalize cross peak intensities. The NMR tubes were then placed in an $800 \mathrm{MHz}$ NMR spectrometer pre-equilibrated at $45^{\circ} \mathrm{C}$. The buffer exchanges, sample transfer, and NMR tube placement were accomplished within $2 \mathrm{~h}$.

After $20-30 \mathrm{~min},{ }^{1} \mathrm{H},{ }^{15} \mathrm{~N}-\mathrm{TROSY} \mathrm{HSQC}^{89-91}$ (for U- ${ }^{15} \mathrm{~N}-\mathrm{C} 99$ at $0.39 \mathrm{mM}$ ) or SOFAST HMQC ${ }^{92,93}$ (for GLVT- ${ }^{15} \mathrm{~N}-\mathrm{C} 99$ at $0.25 \mathrm{mM}$ ) NMR spectra were recorded continuously in the beginning so as to be able to monitor fast-exchanging residues and then once every several days to monitor slow-exchanging residues. Although the cross peaks for most residues in the TM helix of C99 could be located in the TROSY HSQC spectra, those for some glycine, leucine, valine, and threonine residues in $\mathrm{C} 99$ were partially overlapped with signals from imidazole or other residues. For the TM domain specifically, TROSY HSQC spectra on $\mathrm{U}-{ }^{15} \mathrm{~N}-\mathrm{C} 99$ were used for monitoring the amides of M35, A42, I45, I47, and M51 only. To fully resolve the cross peaks for the remaining glycine, leucine, valine, and threonine residues, we employed GLVT- ${ }^{15} \mathrm{~N}-\mathrm{C} 99$ and the SOFAST HMQC pulse program.

All samples were kept at $45{ }^{\circ} \mathrm{C}$ after the first spectrum was recorded. Each acquisition took $1 \mathrm{~h}$ with 16 scans each per 200 increments for TROSY HSQC and $0.5 \mathrm{~h}$ with 48 scans each per 100 increments for SOFAST HMQC. Changes in cross peak intensities were monitored to measure the rate constants. Cross peak intensities of exchangeable amides in the TM helix of $\mathrm{C} 99$ for all time points were normalized relative to the external standard $\left({ }^{15} \mathrm{~N}\right.$-labeled $\mathrm{N}$ acetylglycine). For each residue, a further normalization of its cross peak intensities was made using the first data point for the H-to-D exchange and the last data point for the $\mathrm{D}$-to- $\mathrm{H}$ exchange. The normalized cross peak intensities at two nearby time points were then averaged, i.e., $y_{1}=\left(I_{1}+I_{2}\right) / 2, y_{2}=\left(I_{3}+I_{4}\right) / 2, y_{3}=\left(I_{5}+I_{6}\right) / 2$, etc., for all residues but the fastest-exchanging residues, L34, M35, and G38, and the slowest-exchanging residues, V44 and T48. Since the half-lives of the amide H-to-D and D-to-H exchange processes for L34, M35, and G38 were on the order of magnitude of only $\sim 1 \mathrm{~h}$, we did not average their peak intensities at nearby time points to avoid affecting the time resolution of their exchanges. Since the half-lives of the amide H-to-D and D-to-H exchanges for T44 and T48 were more than 800 $\mathrm{h}$, their normalized cross peak intensities at time points collected within $\sim 10 \mathrm{~h}$ were averaged. For D-to-H exchange, the cross peak intensities for fast-exchanging residues L34, M35, and G38 reached their plateaus within 1 day. After that, although no D-to-H exchange took place for these residues, their cross peak intensities fluctuated within the range of experimental errors in time until the all data collection was completed $\sim 1$ month later. Therefore, if $t_{0}$ are the time points at which they reached their plateaus, we truncated their D-to-H exchange plots at $2 t_{0}$ when we fit the plots to derive their $k(\mathrm{D} \rightarrow \mathrm{H})$ values. The purpose of data averaging and $\mathrm{D}$-to- $\mathrm{H}$ exchange plot truncations was to reduce the fitting error while keeping the change of cross peak intensities during exchanges unaffected. Of the 23 residues in the TM helix of C99, residues 30-33 at the N-terminal region underwent $\mathrm{H} / \mathrm{D}$ exchange that was too fast for us to measure. At the other extreme, we could observe no H/D exchange for residues I45, V46, or I47 over the course of several weeks. The cross peak for I41 was highly overlapped with that for V50 in the TROSY HSQC spectra for $\mathrm{U}_{-}{ }^{15} \mathrm{~N}-\mathrm{C} 99$ and did not show up in the SOFAST HMQC spectra for GLVT- $-{ }^{15} \mathrm{~N}-\mathrm{C} 99$. Therefore, we could not obtain the H/D exchange rate constants for this residue, either. For the remaining 15 residues, we could observe $H / D$ exchange in both directions or at least in the H-to-D exchange direction. Their measurable rate constants were obtained by fitting the plots of the normalized and averaged cross peak intensities against time. For H-to-D exchanges, the equation for fitting was

$$
y=a \exp \left\{-k(\mathrm{H} \rightarrow \mathrm{D})^{*} t\right\}
$$

where $t$ is time, $a$ is a constant, and $k(\mathrm{H} \rightarrow \mathrm{D})^{*}$ is the apparent rate constant and equals the product of $k(\mathrm{H} \rightarrow \mathrm{D})$ and the concentration of deuterons in water $\left([\mathrm{D}]_{\mathrm{w}}\right)$. For $\mathrm{D}$-to- $\mathrm{H}$ exchange, the equation for fitting was

$$
y=-b \exp \left\{-k(\mathrm{D} \rightarrow \mathrm{H})^{*} t\right\}+c
$$

where $b$ is a constant, $c=y$ at $t=+\infty$, and $k(\mathrm{D} \rightarrow \mathrm{H}) *$ is the apparent rate constant and equals the product of $k(\mathrm{D} \rightarrow \mathrm{H})$ and the concentration of protons in water $\left([\mathrm{H}]_{\mathrm{w}}\right)$. $[\mathrm{D}]_{\mathrm{w}}$ for the H-to-D exchanges and $[\mathrm{H}]_{\mathrm{w}}$ for the D-to-H exchanges were $96-98 \%$ and $90 \%$ of the concentration of water hydrogens $(110 \mathrm{M})$, respectively. The $\mathrm{H}$ to-D and $\mathrm{D}$-to-H exchange experiments were accomplished within about 2 and 4 weeks, respectively. For each residue, the $\phi$-value was calculated as $\phi=k(\mathrm{H} \rightarrow \mathrm{D}) / k(\mathrm{D} \rightarrow \mathrm{H})$, and the averaged $\phi$-value from the duplicates is reported here. The uncertainty of each $\phi$-value is half the difference between the $\phi$-values from the duplicates.

Analysis of Backbone Hydrogen Bond Geometry. The ensemble of solution state NMR structures in PDB 2LP1 for C99 in LMPG micelles was used for this analysis. It should be clarified that, while the original experimental studies leading to this ensemble employed full-length C99, both the N-terminus and C-terminus of C99 were seen to be largely disordered, such that coordinates were reported in the PDB file only for the relatively well ordered transmembrane and juxtamembrane segments encompassed by residues $12-57$ in C99 numbering, corresponding to residues 683728 in full-length amyloid precursor protein numbering. The geometry of backbone hydrogen bonds in each model of the solution-state NMR structure of $\mathrm{C} 99$ was analyzed from coordinates of the relevant atoms. The distance between any two arbitrary atoms $\mathrm{A}$ and $\mathrm{B}\left(d_{\mathrm{AB}}\right)$ was calculated as

$$
d_{\mathrm{AB}}=\left[\left(x_{\mathrm{A}}-x_{\mathrm{B}}\right)^{2}+\left(y_{\mathrm{A}}-y_{\mathrm{B}}\right)^{2}+\left(z_{\mathrm{A}}-z_{\mathrm{B}}\right)^{2}\right]^{1 / 2}
$$

where $\left(x_{\mathrm{A}}, y_{\mathrm{A}}, z_{\mathrm{A}}\right)$ and $\left(x_{\mathrm{B}}, y_{\mathrm{B}}, z_{\mathrm{B}}\right)$ are the coordinates of atoms $\mathrm{A}$ and $\mathrm{B}$, respectively. The angle formed by any two arbitrary adjacent bonds $\mathrm{A}-\mathrm{B}$ and $\mathrm{B}-\mathrm{C}\left(\theta_{\mathrm{ABC}}\right)$ was calculated as 


$$
\theta_{\mathrm{ABC}}=\left(180^{\circ} / \pi\right) \arccos \left[\left(d_{\mathrm{AB}}{ }^{2}+d_{\mathrm{BC}}{ }^{2}-d_{\mathrm{AC}}{ }^{2}\right) /\left(2 d_{\mathrm{AB}} d_{\mathrm{BC}}\right)\right]
$$

where the multiplier $180^{\circ} / \pi$ was used to convert the unit of angles from radians to degrees.

Since the amide NH group of each residue in the TM helix of C99 can potentially form either $i, i+3$ or $i, i+4$ backbone hydrogen bonds with the carbonyl groups of their preceding residues, ${ }^{94}$ we determined which type of hydrogen bond the $\mathrm{NH}$ group was more highly populated in, and used that for the geometry calculations. To determine the major type of backbone hydrogen bond, we first calculated the backbone $\mathrm{N}-\mathrm{O}$ distances $\left(d_{\mathrm{NO}}\right)$ and the $\mathrm{NHO}$ angles $\left(\theta_{\mathrm{NHO}}\right)$ for each residue in every model for both $i, i+3$ and $i, i+4$ hydrogen bonds. If $\theta_{\mathrm{NHO}}$ is smaller than $100^{\circ}$ and/or $d_{\mathrm{NO}}$ is larger than $3.5 \AA$ for any type of hydrogen bonds, that type of hydrogen bond was considered not formed. If one type of hydrogen bond was not formed, the other type was considered to be the major type of hydrogen bond if it was formed, and otherwise no hydrogen bond was formed. We also used these criteria to check the possibility of the formation of $i, i+5$ backbone hydrogen bonds, but found none. Next, if both $i, i+3$ and $i, i$ +4 backbone hydrogen bonds were considered formed by the amide $\mathrm{NH}$ group of a residue in a model, we calculated $d_{\mathrm{HO}} \sin \left[\left(180^{\circ}-\right.\right.$ $\left.\left.\theta_{\mathrm{NHO}}\right)\left(\pi / 180^{\circ}\right)\right]$ as illustrated in Scheme 1 , where $d_{\mathrm{HO}}$ is the $\mathrm{H}-\mathrm{O}$

Scheme 1. Calculation Used To Determine the Major Types of Backbone Hydrogen Bonds if the Amide NH Group of a Residue Forms Both $i, i+3$ and $i, i+4$ Backbone Hydrogen Bonds

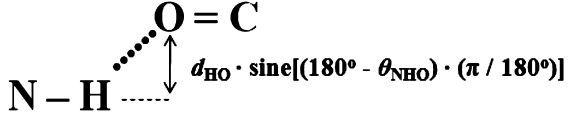

distance, and the multiplier $\pi / 180^{\circ}$ was used to convert the unit of angles from degrees to radians, for both the $i, i+3$ and $i, i+4$ distances. This product represents the deviation of the carbonyl oxygen from the extension of the $\mathrm{N}-\mathrm{H}$ bond. Smaller deviation means larger overlap between the orbitals of $\mathrm{H}$ and $\mathrm{O}$, and vice versa. Therefore, for any residue in any model, the type of backbone hydrogen bond that has the smaller deviation of the carbonyl oxygen from the extension of the $\mathrm{N}-\mathrm{H}$ bond was considered to be the major type of backbone hydrogen bond.

\section{RESULTS AND DISCUSSION}

To obtain well-resolved peaks for all the amide protons, we employed both uniformly ${ }^{15} \mathrm{~N}$-labeled C99 (U- ${ }^{15} \mathrm{~N}-\mathrm{C} 99$ ) and C99 in which only G, L, V, and T residues were labeled with ${ }^{15} \mathrm{~N}$ (GLVT- $\left.{ }^{15} \mathrm{~N}-\mathrm{C} 99\right)$. Spectra were recorded in lysomyristoylphosphatidylglycerol (LMPG) micelles at a LMPGto-C99 molar ratio of more than 500/1. Indeed, no more than one ${ }^{1} \mathrm{H}-{ }^{15} \mathrm{~N}$ cross peak was found for the backbone $\mathrm{NH}$ group of each residue in the ${ }^{1} \mathrm{H},{ }^{15} \mathrm{~N}$-TROSY HSQC $\mathrm{C}^{89-91}$ and the SOFAST HMQC ${ }^{92,93}$ spectra, and each cross peak appeared in the same positions as found previously. ${ }^{72,73}$ These observations indicate that under the conditions of this work C99 still exists as a single, monomeric state.

To measure the amide $\mathrm{H}$-to- $\mathrm{D}$ and $\mathrm{D}$-to- $\mathrm{H}$ exchange rate constants, purified and concentrated U- ${ }^{15} \mathrm{~N}-\mathrm{C} 99$ or GLVT- ${ }^{15} \mathrm{~N}$ C99 samples were buffer-exchanged from $\mathrm{H}_{2} \mathrm{O}(95 \%(\mathrm{v} / \mathrm{v}))$ to $\mathrm{D}_{2} \mathrm{O}(96-98 \%(\mathrm{v} / \mathrm{v}))$ then from $\mathrm{D}_{2} \mathrm{O}(96-98 \%(\mathrm{v} / \mathrm{v}))$ to $\mathrm{H}_{2} \mathrm{O}$ $(90 \%(\mathrm{v} / \mathrm{v})) .{ }^{1} \mathrm{H},{ }^{15} \mathrm{~N}$-TROSY HSQC or SOFAST HMQC spectra were then serially recorded. Because of problems with peak overlap, we collected and analyzed data both for uniformly ${ }^{15} \mathrm{~N}$-labeled C99 and for C99 that was only GLVT- ${ }^{15} \mathrm{~N}$-labeled. For the $\mathrm{H}$-to- $\mathrm{D}$ and $\mathrm{D}$-to-H exchange reactions, we monitored the disappearance or recovery of amide ${ }^{1} \mathrm{H}-{ }^{15} \mathrm{~N}$ cross peaks for the TM helix of C99 relative to the cross peak for the standard
${ }^{15} \mathrm{~N}$-labeled $\mathrm{N}$-acetylglycine over time. This standard was physically isolated from the protein samples using an NMR tube insert (see Materials and Methods). The $k(\mathrm{H} \rightarrow \mathrm{D})$ values for the exchangeable residues were found to vary over 3 orders of magnitude (Figure 2, Table S1, and Figure S1A-C),
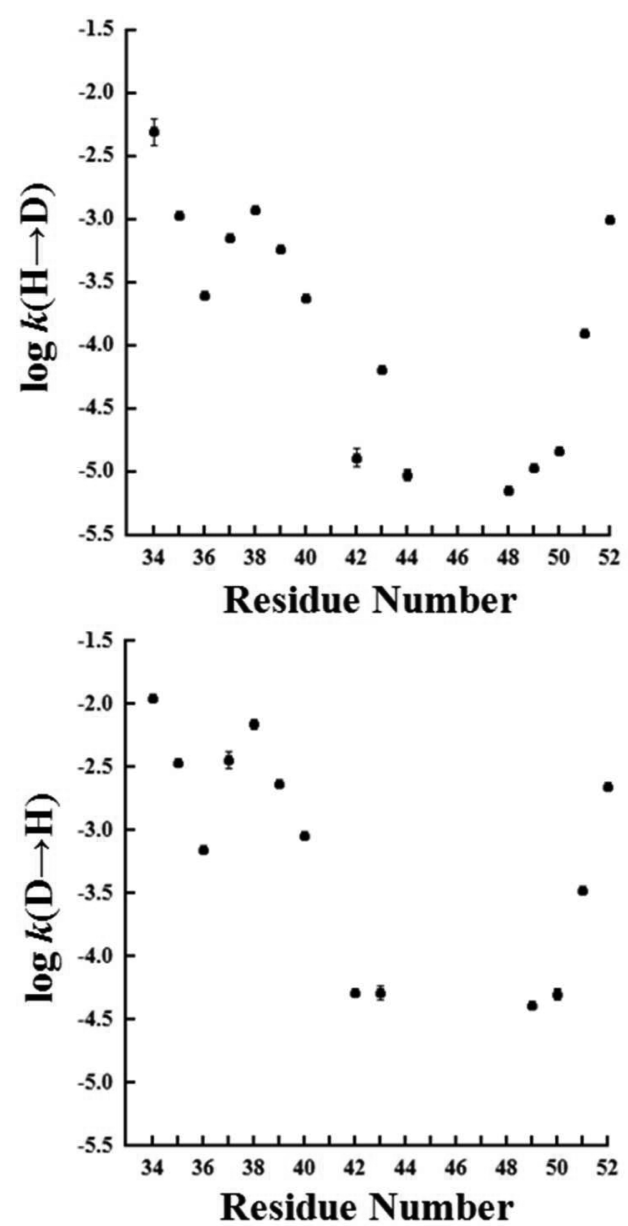

Figure 2. Amide H/D exchange rate constants for the transmembrane segment of C99. Upper: Amide H-to-D exchange rates constant. Lower: Amide D-to-H exchange rate constants. The exchange rate constants in the logarithm are in units of $\mathrm{M}^{-1} \cdot \mathrm{hr}^{-1}$. Error bars represent the discrepancy between two replicates. Only two error bars are obvious in each plot, because the error bars for other data points are smaller than the size of the data points. Positions without data points are sites where exchange was too slow to measure (residues 45-47 for the H-to-D exchange and residues $44-48$ for the D-to-H exchange) or could not be measured for technical reasons (residue 41).

corresponding to half-lives ranging from a few hours to several weeks. These results are comparable to prior rough measurements of the H-to-D exchange rate constants for amides in this helix. ${ }^{73}$ However, in those measurements $k(\mathrm{H} \rightarrow \mathrm{D})$ for each amide was determined from the cross peak intensities at only two time points.

Of the 23 residues in the TM helix of C99, we were able to obtain $\phi$-values for 15 amide protons. Among these 15 sites, amide protons at residues 34-40, 42, 43, and 49-52 were found to be completely exchangeable, with measurable rate constants in both the H-to-D and D-to-H exchange directions (Figure 2, Table S1, and Figure S1A-C,E-G)). For two residues, V44 and T48, we could only obtain a lower limit for 
the $\phi$-values. For the V44 and T48 amides, we obtained the $k(\mathrm{H} \rightarrow \mathrm{D})$ values by fitting their slow $\mathrm{H}$-to-D exchange time traces, but we could not measure the $k(\mathrm{D} \rightarrow \mathrm{H})$ values from their D-to-H exchange time traces because the back exchange reactions were too slow (Figure 2, Table S1, and Figure $\mathrm{S} 1 \mathrm{C}, \mathrm{H}))$. Nevertheless, we can conclude that the $\phi$-values for V44 and T48 are clearly greater than 1, indicating that these hydrogen bonds are very weak. For the remaining eight residues, the $\mathrm{H}$-to- $\mathrm{D}$ and $\mathrm{D}$-to- $\mathrm{H}$ exchange rate constants were either too fast or too slow to measure (Figure S1D,H) or could not be measured accurately due to spectral overlaps (see Materials and Methods). From our previously determined scale factor, $\mathrm{SF}=\partial\left(\Delta G^{\mathrm{HB}}\right) / \partial(R T \ln \phi)=-7.0 \pm 0.7$, we could convert the measured $\phi$-values into $\Delta G^{\mathrm{HB}}$ (relative to the water-to-water hydrogen bond). The final $\phi$ and $\Delta G^{\mathrm{HB}}$ values for the 15 measurable hydrogen bonds are summarized in Figure 3 and Table S1.

We observed a wide variation in hydrogen bond strengths. Most of the hydrogen bonds in the C99 TM helix are much stronger than water-water hydrogen bonds, some by more than $6 \mathrm{kcal} / \mathrm{mol}$, indicating that backbone hydrogen bonds can be extremely strong. Remarkably, however, some hydrogen
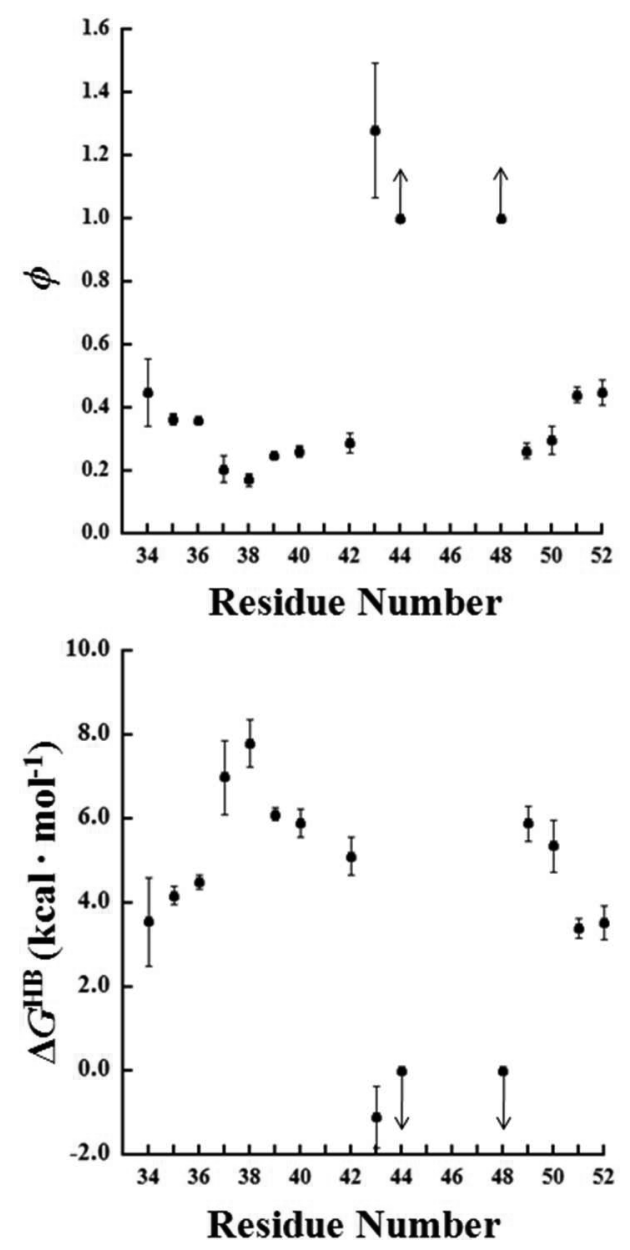

Figure 3. Hydrogen bond strengths in the transmembrane segment of C99. $\phi$-values are shown in the upper plot, and the corresponding $\Delta G^{\mathrm{HB}}$ values are shown in the lower plot for hydrogen bonds formed by the amide $\mathrm{NH}$ groups in the TM helix of C99. The arrows indicate that the $\phi$-values and $\Delta G^{\mathrm{HB}}$ for both residues 44 and 48 are above 1 and below $0 \mathrm{kcal} / \mathrm{mol}$, respectively. Error bars represent the discrepancy of the results in two replicates. bonds-at residues T43, V44, and T48-are even weaker than water-water hydrogen bonds. Counterintuitively, the weakest hydrogen bonds are found at positions where exchange rates are slow, suggesting that their environments (e.g. water accessibility, local $\mathrm{pH}$ ) are simply unfavorable for exchange. These results reveal that backbone hydrogen bond strengths in a TM helix can be far from uniform.

Given the large variation in hydrogen bond strengths we wondered whether there was any influence on hydrogen bond geometry in the helix. We therefore examined the $\mathrm{N}-\mathrm{O}$ distances for the backbone hydrogen bonds by analyzing the 30 models of the backbone structure of C99 consistent with solution-state NMR constraints (PDB code: 2LP1). Figure 4

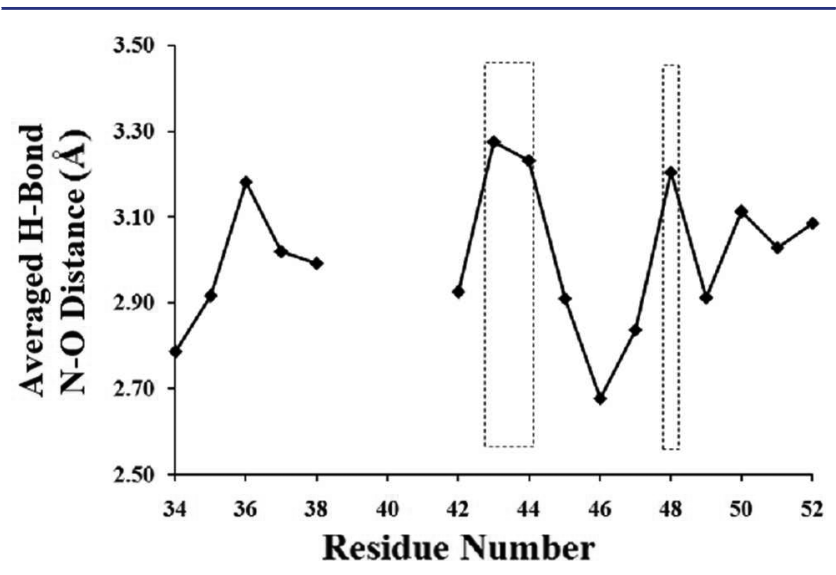

Figure 4. Average $\mathrm{N}-\mathrm{O}$ distance of the backbone hydrogen bonds in the transmembrane segment of $\mathrm{C} 99$. The value in the vertical axis is the $\mathrm{N}-\mathrm{O}$ distance averaged from the 30 models of the solution-state NMR structure of C99 (PDB code: 2LP1). Rectangular boxes are drawn to indicate the local maxima found at residues 43,44 , and 48 . Results for residues 39-41 are not included in this plot, because their amide $\mathrm{NH}$ groups form backbone hydrogen bonds in less than 10 out of the 30 models and their averaged $d_{\mathrm{NO}}$ values from the 30 models are also all above $3.5 \AA$, which is beyond our threshold for hydrogen bond formation.

shows the averaged $\mathrm{N}-\mathrm{O}$ distances $\left(d_{\mathrm{NO}}\right)$ for backbone hydrogen bonds that form in 10 or more models against the residue number each amide $\mathrm{NH}$ group. The average of $d_{\mathrm{NO}}$ was taken from all the 30 models. Figure S2 shows the distances for each individual model.

For residues immediately after the diglycine hinge, ${ }^{72}$ backbone hydrogen bonds to the $\mathrm{NH}$ group of V39, V40, and I41 were observed in less than 10 models, and their average $d_{\text {NO }}$ values from the 30 models are above $3.5 \AA$. The existence of the helix kink centering the diglycine hinge near residues 39-41 makes it impossible for them to find a hydrogen-bond acceptor in the backbone of the same helix (see Figure 1). Surprisingly, however, the $\phi$-values for V39 and V40 are low, indicating that these two residues make strong hydrogen bonds. It seems possible that unseen water molecules manage to insert at these positions to satisfy the hydrogen bonding potential. Perhaps the glycine residues are particularly favorable places for water to penetrate. Indeed, the plots of exchange rates against residue numbers in Figure 2 show a local maximum around the G37G38 diglycine hinge, which suggests that this region has a higher water accessibility than nearby residues. We also note that the $\mathrm{N}$-terminal region shows high exchange rates, indicative of water penetration and flexibility and also low $\phi$ values. The low $\phi$-values may result from hydrogen bonds not 
only within the backbone but also between backbone $\mathrm{N}-\mathrm{H}$ moieties and water or detergent head groups.

Although the remaining residues in the TM helix of C99 form hydrogen bonds as expected, the averaged $d_{\mathrm{NO}}$ values have local maxima at T43, V44, and T48. This indicates that the NMR constraints are remarkably consistent with weak amide hydrogen bonding at T43, V44, and T48 allowing them to stretch more readily than other residues in the TM helix.

The T48 amide $\mathrm{NH}$ group is one residue upstream of the $\varepsilon 48$ cut site (one of the initial $\gamma$-secretase cut sites), and the $\mathrm{T} 43$ and V44 amide $\mathrm{NH}$ groups are exactly at the $\gamma 42$ and $\gamma 43$ cut sites (two of the final $\gamma$-secretase cut sites). We were unable to experimentally determine strengths of the hydrogen bonds in between these sites. Our findings of the low $\phi$ and high $d_{\mathrm{NO}}$ values for the $\mathrm{NH}$ groups of T43, V44, and T48 suggest that their backbone hydrogen bonds are weak. Since a helix-to-coil transition is required for the cleavage of the TM helix in C99 by $\gamma$-secretase, ${ }^{62}$ it suggests that the local weak points created at T43, V44, and T48 may enhance the preference of helix-to-coil transitions near or at these residues and may facilitate the selection of cut sites by $\gamma$-secretase. Indeed, it was previously observed that this helix adopts a mixture of helical and nonhelical conformations at T43 (as also reported for the kink and termini) in vesicles using a neuronal lipid mixture at $37^{\circ} \mathrm{C}$. ${ }^{76}$ Our results not only agree with the finding of flexibility at this residue but also provide a physical explanation for it.

\section{CONCLUSION}

To our knowledge, our results provide the first measurements of backbone hydrogen bond strengths in transmembrane helices. Most of the hydrogen bonds are much stronger than water-water hydrogen bonds by at least $3 \mathrm{kcal} / \mathrm{mol}$ (Figure 3 ) as might be expected for hydrogen bonds in an apolar environment. ${ }^{22,95,96}$ Moreover, our measurements are made in detergent micelles, where water accessibility and helix dynamics are likely to be even higher than in true bilayers. These results contrast greatly with experimental measurements of side-chain hydrogen bonds in membrane proteins, which have generally be found to be rather weak. ${ }^{1-10}$ Side chains have considerable flexibility, however, and can form many alternative, competitive hydrogen bonds with other polar groups or water. ${ }^{1}$ In the case of backbone hydrogen bonds, however, choices for alternative hydrogen bonds are generally limited, particularly in an isolated TM helix like in C99. Moreover, there are cooperative avidity effects due to neighboring hydrogen bonds in a TM helix that would strengthen individual hydrogen bonds in the helix.

Strong hydrogen bonds might create rigid helices, yet TM helices must bend and flex during functional cycles. We had previously proposed a generic mechanism for allowing TM helix flexibility by shifting backbone hydrogen bond partners. ${ }^{19}$ Here we suggest additional more specific mechanisms for allowing flexibility that may also operate. In particular, we suggest that the kinked conformation of the diglycine hinge is stabilized by hydrogen bonding to cryptic water molecules. It is known that the TM helix of C99 can shift between the kinked and straight conformations when it is solubilized in different lipid environments. ${ }^{97}$ The penetration of water molecules at the diglycine hinge provides a mechanism for allowing kinking without the loss of hydrogen bonds. ${ }^{17}$ Our results also suggest that the TM helix sequence can encode weaker backbone hydrogen bonds at specific locations, generating possible locations for more facile helix distortions. The fact that it is possible to encode hydrogen bond strength variation in a TM helix suggest that hydrogen bond strength variation could be utilized by evolution to provide functional information, i.e., sites that facilitate membrane protein dynamics or in the case of C99, perhaps cleavage site preferences. How sequences can encode hydrogen bond strength variations in TM helices is an interesting potential area of investigation. In this regard, it is noteworthy that T43 and T48, where weak backbone amide hydrogen bonds occur, are the only two hydrophilic residues in the TM helix.

\section{ASSOCIATED CONTENT}

\section{S Supporting Information}

The Supporting Information is available free of charge on the ACS Publications website at DOI: 10.1021 /jacs.7b04819.

Additional data are shown in Table S1, Figure S1, and Figure S2 (PDF)

\section{AUTHOR INFORMATION}

\section{Corresponding Author}

*bowie@mbi.ucla.edu

ORCID

James U. Bowie: 0000-0003-2249-5908

Present Address

${ }^{\S}$ Z.C.: Beijing Abace-Biology Co., Ltd., Beijing 100176, China

Notes

The authors declare no competing financial interest.

\section{ACKNOWLEDGMENTS}

This work was supported by U.S. NIH grants R01 GM063919 to J.U.B., R01 GM106672 and AG056147 to C.R.S., and T32 CA009582 to J.M.H. We thank Dr. Robert Peterson for technical support and assistance with the NMR experiments.

\section{REFERENCES}

(1) Bowie, J. U. Curr. Opin. Struct. Biol. 2011, 21 (1), 42-49.

(2) Choma, C.; Gratkowski, H.; Lear, J. D.; DeGrado, W. F. Nat. Struct. Biol. 2000, 7 (2), 161-166.

(3) Zhou, F. X.; Merianos, H. J.; Brunger, A. T.; Engelman, D. M. Proc. Natl. Acad. Sci. U. S. A. 2001, 98 (5), 2250-2255.

(4) Gratkowski, H.; Lear, J. D.; DeGrado, W. F. Proc. Natl. Acad. Sci. U. S. A. 2001, 98 (3), 880-885.

(5) Fleming, K. G.; Engelman, D. M. Proc. Natl. Acad. Sci. U. S. A. 2001, 98 (25), 14340-14344.

(6) Zhou, F. X.; Cocco, M. J.; Russ, W. P.; Brunger, A. T.; Engelman, D. M. Nat. Struct. Biol. 2000, 7 (2), 154-160.

(7) Stanley, A. M.; Fleming, K. G. J. Mol. Biol. 2007, 370 (5), 912924.

(8) Li, E.; You, M.; Hristova, K. J. Mol. Biol. 2006, 356 (3), 600-612.

(9) Joh, N. H.; Min, A.; Faham, S.; Whitelegge, J. P.; Yang, D.; Woods, V. L.; Bowie, J. U. Nature 2008, 453 (7199), 1266-1270.

(10) Hong, H.; Blois, T. M.; Cao, Z.; Bowie, J. U. Proc. Natl. Acad. Sci. U. S. A. 2010, 107 (46), 19802-19807.

(11) Hall, S. E.; Roberts, K.; Vaidehi, N. J. Mol. Graphics Modell. 2009, 27, 944-950.

(12) Langelaan, D. N.; Wieczorek, M.; Blouin, C.; Rainey, J. K. J. Chem. Inf. Model. 2010, 50 (12), 2213-2220.

(13) Meruelo, A. D.; Samish, I.; Bowie, J. U. Protein Sci. 2011, 20 (7), 1256-1264.

(14) Bright, J. N.; Shrivastava, I. H.; Cordes, F. S.; Sansom, M. S. P. Biopolymers 2002, 64 (6), 303-313.

(15) Shi, L.; Liapakis, G.; Xu, R.; Guarnieri, F.; Ballesteros, J. A.; Javitch, J. A. J. Biol. Chem. 2002, 277, 40989-40996.

(16) Yohannan, S.; Faham, S.; Yang, D.; Whitelegge, J. P.; Bowie, J. U. Proc. Natl. Acad. Sci. U. S. A. 2004, 101 (4), 959-963. 
(17) Miyano, M.; Ago, H.; Saino, H.; Hori, T.; Ida, K. Curr. Opin. Struct. Biol. 2010, 20 (4), 456-463.

(18) Wigley, W. C.; Corboy, M. J.; Cutler, T. D.; Thibodeau, P. H.; Oldan, J.; Lee, M. G.; Rizo, J.; Hunt, J. F.; Thomas, P. J. Nat. Struct. Biol. 2002, 9, 381-388.

(19) Cao, Z.; Bowie, J. U. Proc. Natl. Acad. Sci. U. S. A. 2012, 109 (21), 8121-8126.

(20) Fersht, A. R.; Shi, J.-P.; Knill-Jones, J.; Lowe, D. M.; Wilkinson, A. J.; Blow, D. M.; Brick, P.; Carter, P.; Waye, M. M. Y.; Winter, G. Nature 1985, 314 (6008), 235-238.

(21) Fersht, A. R.; Matouschek, A.; Serrano, L. J. Mol. Biol. 1992, 224 (3), 771-782.

(22) Rose, G. D.; Wolfenden, R. Annu. Rev. Biophys. Biomol. Struct. 1993, 22 (1), 381-415.

(23) Shirley, B. A.; Stanssens, P.; Hahn, U.; Pace, C. N. Biochemistry 1992, 31 (3), 725-732.

(24) Marqusee, S.; Sauer, R. T. Protein Sci. 1994, 3 (12), 2217-2225.

(25) Myers, J. K.; Pace, C. N. Biophys. J. 1996, 71 (4), 2033-2039.

(26) Myers, J. K.; Oas, T. G. Biochemistry 1999, 38 (21), 6761-6768.

(27) Takano, K.; Yamagata, Y.; Funahashi, J.; Hioki, Y.; Kuramitsu, S.; Yutani, K. Biochemistry 1999, 38 (39), 12698-12708.

(28) Fernández-Recio, J.; Romero, A.; Sancho, J. J. Mol. Biol. 1999, 290 (1), 319-330.

(29) Albeck, S.; Unger, R.; Schreiber, G. J. Mol. Biol. 2000, 298 (3), 503-520.

(30) Pace, C. N.; Horn, G.; Hebert, E. J.; Bechert, J.; Shaw, K.; Urbanikova, L.; Scholtz, J. M.; Sevcik, J. J. Mol. Biol. 2001, 312 (2), 393-404.

(31) Takano, K.; Scholtz, J. M.; Sacchettini, J. C.; Pace, C. N. J. Biol. Chem. 2003, 278 (34), 31790-31795.

(32) Jang, D. S.; Cha, H. J.; Cha, S.-S.; Hong, B. H.; Ha, N.-C.; Lee, J. Y.; Oh, B.-H.; Lee, H.-S.; Choi, K. Y. Biochem. J. 2004, 382 (3), 967.

(33) Campos, L. A.; Cuesta-López, S.; López-Llano, J.; Falo, F.; Sancho, J. Biophys. J. 2005, 88 (2), 1311-1321.

(34) Pace, C. N. Nat. Struct. Mol. Biol. 2009, 16 (7), 681-682.

(35) Wang, M.; Wales, T. E.; Fitzgerald, M. C. Proc. Natl. Acad. Sci. U. S. A. 2006, 103 (8), 2600-2604.

(36) Fu, Y.; Gao, J.; Bieschke, J.; Dendle, M. A.; Kelly, J. W. J. Am. Chem. Soc. 2006, 128 (50), 15948-15949.

(37) Gao, J.; Kelly, J. W. Protein Sci. 2008, 17 (6), 1096-1101.

(38) Gao, J.; Bosco, D. A.; Powers, E. T.; Kelly, J. W. Nat. Struct. Mol. Biol. 2009, 16 (7), 684-690.

(39) Cao, Z.; Bowie, J. U. Protein Sci. 2014, 23 (5), 566-575.

(40) Shi, Z.; Krantz, B. A.; Kallenbach, N.; Sosnick, T. R. Biochemistry 2002, 41 (7), 2120-2129.

(41) Hibbert, F.; Emsley, J. In Advances in Physical Organic Chemistry; Bethell, D., Ed.; Academic Press: San Diego,1991; Vol. 26, pp 255379.

(42) Vakonakis, I.; Salazar, M.; Kang, M.; Dunbar, K. R.; LiWang, A. C. J. Biomol. NMR 2003, 25 (2), 105-112.

(43) Loh, S. N.; Markley, J. L. Biochemistry 1994, 33 (4), 1029-1036.

(44) Bowers, P. M.; Klevit, R. E. Nat. Struct. Mol. Biol. 1996, 3 (6), $522-531$.

(45) LiWang, A. C.; Bax, A. J. Am. Chem. Soc. 1996, 118 (50), 12864-12865.

(46) Khare, D.; Alexander, P.; Orban, J. Biochemistry 1999, 38 (13), 3918-3925.

(47) Halkides, C. J.; Wu, Y. Q.; Murray, C. J. Biochemistry 1996, 35 (49), 15941-15948.

(48) Harris, T. K.; Abeygunawardana, C.; Mildvan, A. S. Biochemistry 1997, 36 (48), 14661-14675.

(49) Lin, J.; Westler, W. M.; Cleland, W. W.; Markley, J. L.; Frey, P. A. Proc. Natl. Acad. Sci. U. S. A. 1998, 95 (25), 14664-14668.

(50) Markley, J. L.; Westler, W. M. Biochemistry 1996, 35 (34), 11092-11097.

(51) Takeda, M.; Jee, J.; Terauchi, T.; Kainosho, M. J. Am. Chem. Soc. 2010, 132 (17), 6254-6260.

(52) Edison, A. S.; Weinhold, F.; Markley, J. L. J. Am. Chem. Soc. 1995, 117 (38), 9619-9624.
(53) Gregory, G. C.; Halliday, G. M. Neurotoxic. Res. 2005, 7 (1-2), 29-41.

(54) Hardy, J.; Selkoe, D. J. Science 2002, 297 (5580), 353-356.

(55) Haass, C.; Selkoe, D. J. Nat. Rev. Mol. Cell Biol. 2007, 8 (2), 101-112.

(56) Lichtenthaler, S. F.; Haass, C.; Steiner, H. J. Neurochem. 2011, 117 (5), 779-796.

(57) Kaden, D.; Munter, L. M.; Reif, B.; Multhaup, G. Eur. J. Cell Biol. 2012, 91 (4), 234-239.

(58) Beel, A. J.; Mobley, C. K.; Kim, H. J.; Tian, F.; Hadziselimovic, A.; Jap, B.; Prestegard, J. H.; Sanders, C. R. Biochemistry 2008, 47 (36), 9428-9446.

(59) Song, Y.; Hustedt, E. J.; Brandon, S.; Sanders, C. R. Biochemistry 2013, 52 (30), 5051-5064.

(60) Wang, H.; Barreyro, L.; Provasi, D.; Djemil, I.; Torres-Arancivia, C.; Filizola, M.; Ubarretxena-Belandia, I. J. Mol. Biol. 2011, 408 (5), 879-895.

(61) Munter, L.-M.; Voigt, P.; Harmeier, A.; Kaden, D.; Gottschalk, K. E.; Weise, C.; Pipkorn, R.; Schaefer, M.; Langosch, D.; Multhaup, G. EMBO J. 2007, 26 (6), 1702-1712.

(62) Sato, T.; Tang, T.-C.; Reubins, G.; Fei, J. Z.; Fujimoto, T.; Kienlen-Campard, P.; Constantinescu, S. N.; Octave, J.-N.; Aimoto, S.; Smith, S. O. Proc. Natl. Acad. Sci. U. S. A. 2009, 106 (5), 1421-1426.

(63) Nadezhdin, K. D.; Bocharova, O. V.; Bocharov, E. V.; Arseniev, A. S. FEBS Lett. 2012, 586 (12), 1687-1692.

(64) Chen, W.; Gamache, E.; Rosenman, D. J.; Xie, J.; Lopez, M. M.; Li, Y.-M.; Wang, C. Nat. Commun. 2014, 5, 3037.

(65) Dominguez, L.; Foster, L.; Straub, J. E.; Thirumalai, D. Proc. Natl. Acad. Sci. U. S. A. 2016, 113 (36), E5281-E5287.

(66) Winkler, E.; Julius, A.; Steiner, H.; Langosch, D. Biochemistry 2015, 54 (40), 6149-6152.

(67) Gerber, H.; Wu, F.; Dimitrov, M.; Garcia Osuna, G. M.; Fraering, P. C. J. Biol. Chem. 2017, 292 (9), 3751-3767.

(68) Jung, J. I.; Premraj, S.; Cruz, P. E.; Ladd, T. B.; Kwak, Y.; Koo, E. H.; Felsenstein, K. M.; Golde, T. E.; Ran, Y. PLoS One 2014, 9 (10), e111553.

(69) Fernandez, M. A.; Biette, K. M.; Dolios, G.; Seth, D.; Wang, R.; Wolfe, M. S. Biochemistry 2016, 55 (40), 5675-5688.

(70) Osenkowski, P.; Ye, W.; Wang, R.; Wolfe, M. S.; Selkoe, D. J. J. Biol. Chem. 2008, 283 (33), 22529-22540.

(71) Fukumori, A.; Steiner, H. EMBO J. 2016, 35 (15), 1628-1643.

(72) Barrett, P. J.; Song, Y.; Van Horn, W. D.; Hustedt, E. J.; Schafer, J. M.; Hadziselimovic, A.; Beel, A. J.; Sanders, C. R. Science 2012, 336 (6085), 1168-1171.

(73) Pester, O.; Barrett, P. J.; Hornburg, D.; Hornburg, P.; Pröbstle, R.; Widmaier, S.; Kutzner, C.; Dürrbaum, M.; Kapurniotu, A.; Sanders, C. R.; Scharnagl, C.; Langosch, D. J. Am. Chem. Soc. 2013, 135 (4), 1317-1329.

(74) Scharnagl, C.; Pester, O.; Hornburg, P.; Hornburg, D.; Götz, A.; Langosch, D. Biophys. J. 2014, 106 (6), 1318-1326.

(75) Tyndall, J. D. A.; Nall, T.; Fairlie, D. P. Chem. Rev. 2005, 105 (3), 973-999.

(76) Lu, J.-X.; Yau, W.-M.; Tycko, R. Biophys. J. 2011, 100 (3), 711719.

(77) Osenkowski, P.; Ye, W.; Wang, R.; Wolfe, M. S.; Selkoe, D. J. J. Biol. Chem. 2008, 283 (33), 22529-22540.

(78) Winkler, E.; Kamp, F.; Scheuring, J.; Ebke, A.; Fukumori, A.; Steiner, H. J. Biol. Chem. 2012, 287 (25), 21326-21334.

(79) Holmes, O.; Paturi, S.; Ye, W.; Wolfe, M. S.; Selkoe, D. J. Biochemistry 2012, 51 (17), 3565-3575.

(80) Langosch, D.; Scharnagl, C.; Steiner, H.; Lemberg, M. K. Trends Biochem. Sci. 2015, 40 (6), 318-327.

(81) Elad, N.; De Strooper, B.; Lismont, S.; Hagen, W.; Veugelen, S.; Arimon, M.; Horré, K.; Berezovska, O.; Sachse, C.; Chávez-Gutiérrez, L. J. Cell Sci. 2015, 128 (3), 589-598.

(82) Bai, X. C.; Rajendra, E.; Yang, G.; Shi, Y.; Scheres, S. H. eLife 2015, 4, e11182.

(83) Bolduc, D. M.; Montagna, D. R.; Seghers, M. C.; Wolfe, M. S.; Selkoe, D. J. eLife 2016, 5, e17578. 
(84) Dawson, J. P.; Weinger, J. S.; Engelman, D. M. J. Mol. Biol. 2002, 316 (3), 799-805.

(85) Call, M. E.; Wucherpfennig, K. W. Mol. Immunol. 2004, 40 (18), $1295-1305$.

(86) Senes, A.; Engel, D. E.; DeGrado, W. F. Curr. Opin. Struct. Biol. 2004, 14 (4), 465-479.

(87) White, S. H. Adv. Protein Chem. 2005, 72, 157-172.

(88) Tatko, C. D.; Nanda, V.; Lear, J. D.; Degrado, W. F. J. Am. Chem. Soc. 2006, 128 (13), 4170-4171.

(89) Pervushin, K. V.; Wider, G.; Wüthrich, K. J. Biomol. NMR 1998, 12 (2), 345-348.

(90) Rance, M.; Loria, J. P.; Palmer, A. G. J. Magn. Reson. 1999, 136 (1), 92-101.

(91) Zhu, G.; Kong, X. M.; Sze, K. H. J. Biomol. NMR 1999, 13 (1), $77-81$.

(92) Schanda, P.; Brutscher, B. J. Am. Chem. Soc. 2005, 127 (22), 8014-8015.

(93) Schanda, P.; Kupče, E.; Brutscher, B. J. Biomol. NMR 2005, 33 (4), 199-211.

(94) Barlow, D. J.; Thornton, J. M. J. Mol. Biol. 1988, 201 (3), 601619.

(95) Ben-Tal, N.; Sitkoff, D.; Topol, I. A.; Yang, A.-S.; Burt, S. K.; Honig, B. J. Phys. Chem. B 1997, 101 (3), 450-457.

(96) Mitchell, J. B. O.; Price, S. L. J. Comput. Chem. 1990, 11 (10), $1217-1233$.

(97) Lemmin, T.; Dimitrov, M.; Fraering, P. C.; Dal Peraro, M. J. Biol. Chem. 2014, 289 (10), 6763-6774. 